\title{
AFPep, a novel drug for the prevention and treatment of breast cancer, does not disrupt the estrous cycle or fertility in rats
}

\author{
AMANDA M. TOWER ${ }^{1}$, ANDREA TRINWARD ${ }^{1}$, KATIE LEE $^{1}$, LEROY JOSEPH ${ }^{1}$, \\ HERBERT I. JACOBSON ${ }^{2,3}$, JAMES A. BENNETT ${ }^{2}$ and THOMAS T. ANDERSEN ${ }^{1}$
}

Laboratory for Cancer Control, ${ }^{1}$ Center for Cardiovascular Science; ${ }^{2}$ Center for Immunology and Microbial Disease;

${ }^{3}$ Department of Obstetrics, Gynecology and Reproductive Science, Albany Medical College, Albany, NY 12208, USA

Received December 19, 2008; Accepted January 29, 2009

DOI: $10.3892 /$ or_00000405

\begin{abstract}
Pregnancy lowers the risk of breast cancer, largely attributable to alpha-fetoprotein (AFP). A small AFP-derived peptide (AFPep) which mimics the active site of AFP has been developed and may be useful for decreasing the risk of breast cancer for women. AFPep has been shown previously to stop the growth of estrogen-dependent human breast cancer xenografts in mice and prevent carcinogen-induced breast cancer in a rat model. Since AFPep disrupts an estrogen-responsive pathway, it is essential to assess its effects on the female reproductive cycle and fertility. Ten cycling female Sprague-Dawley rats (age 81 days) were given $100 \mu \mathrm{g}$ AFPep in saline s.c. daily for 20 days. A second group of ten rats was given $50 \mu \mathrm{g}$ tamoxifen s.c. daily and a third group received saline only. Vaginal smears were obtained twice per day and stained to assess estrous cycle phase. After completion of estrous cycle assessment (five cycles, 21 days), rats were maintained on drug and allowed to mate. Effects on birth of offspring and maternal body weights were assessed. AFPep had no significant effect on the incidence or duration of any estrous cycle phase, and no effect on reproductive potential or maternal body mass. Tamoxifen significantly increased the length of diestrus, locking the cycle in this phase for most animals. Only half of the tamoxifen-treated rats mated, and none became pregnant. Tamoxifen significantly slowed the rate of body mass increase. In rats, AFPep has no toxicity and no effect on female reproduction. This molecule may be developed into an attractive modality for prevention of breast cancer in women.
\end{abstract}

\section{Introduction}

With any new drug, especially one with a novel mechanism, it is essential to understand the side effects and toxicity in

Correspondence to: Dr Thomas T. Andersen, Laboratory for Cancer Control MC-8, Albany Medical College, Albany, NY 12208, USA

E-mail: anderst@mail.amc.edu

Key words: alpha-fetoprotein, chemoprevention, estrous cycle, side effects, rat, tamoxifen animal models before entering clinical trials. Most drugs now on the market have unwanted side effects, including tamoxifen, which engenders a low but significant incidence of uterine cancer and side effects including thrombosis, endometriosis, stroke and fertility issues (1-3). A critical objective in the development of chronically administered preventative agents, vis-a-vis therapeutic agents, is to eliminate side effects. Efficacy without toxicity should be the goal of drug development. Peptide drugs offer attractive options because the large number of possible combinations of amino acids can lead to exquisite specificity and hence the desired efficacy, and because metabolites of peptides are simple amino acids without toxicity. The purpose of this study was to assess the effects of a novel peptide, shown to be useful for the treatment and prevention of breast cancer in animal models (4-6), on the estrous cycle and reproductive potential in rats prior to initiation of pre-clinical translational studies.

AFPep is a newly developed peptide demonstrated to have significant activity in the prevention and treatment of breast cancer in experimental models (4-6) and which is being developed for prevention of breast cancer in women. Effective use of this agent may require its administration to premenopausal and postmenopausal women. Drug acceptability by healthy women necessitates negligible toxicity and the absence of side effects. AFPep was derived from alpha-fetoprotein (AFP), a pregnancy-associated protein in maternal plasma secreted by the fetal liver. Epidemiological studies have suggested that AFP is responsible for women's reduced risk of breast cancer following term pregnancy $(7,8)$. These epidemiology studies have been supported by laboratory studies which have shown that AFP inhibits the growth of breast cancer cells in culture and in experimental animals $(9,10)$. However, a large molecule such as AFP would be expected to bear multiple epitopes, and as a drug, to have an array of biological activities, a situation for which ample precedent exists (11-13). In fact, in addition to its antibreast cancer activity, AFP has been shown to have activities associated with immunoregulation (14), hepatic regeneration (15), embryonic tissue differentiation (16) and disruption of the estrous $(17,18)$ and menstrual cycle $(19,20)$.

We used a parsing approach to identify the anti-oncogenic active site of the 590-amino acid AFP, deliberately eliminating any other activity that might be associated with the parent protein. Festin et al (21) located the anti-oncogenic activity to 
Domain III of AFP (22), and Eisele et al and Mizejewski et al reported that a portion of Domain III (a 34-mer peptide) retained the activity (23-25). Mesfin et al (26) further parsed the biological activity to 8 amino acids near the carboxyl end of the 34-mer peptide and DeFreest et al (27) and Kirshner et al (28) showed that the pharmacophore was contained in 5 amino acids within this 8 -mer peptide. Subsequently, Mesfin et al (29) cyclized the short peptide and achieved a dramatic improvement in the dose-response curve and in the stability of the molecule.

AFPep was compared with tamoxifen for activity against growth of human breast cancer xenografts implanted in immune-deficient mice. Both AFPep and tamoxifen inhibited growth of MCF-7 and of T47D human breast cancer xenografts, both of which are estrogen-dependent (i.e., ER+). Neither tamoxifen nor AFPep inhibited growth of estrogenindependent (ER-) human breast cancer MDA-MB-231 and BT-20 xenografts. AFPep completely prevented the growth of a tamoxifen-resistant sub-line of MCF-7 human cancer, suggesting that the peptide inhibits cancer growth by a mechanism different from that of tamoxifen (5). AFPep does not interfere with the binding of $\left[{ }^{3} \mathrm{H}\right]$-estradiol to the estrogen receptor, whereas tamoxifen does. Notably, while enhancing the anti-tumor activity of tamoxifen (4), AFPep reduces the uterotrophic effect of tamoxifen in mice and so averts a major undesirable side effect of tamoxifen (5). AFPep inhibited the appearance of breast tumors in a chemicallyinduced cancer model (6). Treatment of carcinogen-exposed rats with AFPep yielded significantly more tumor-free days, significantly lower tumor incidence, and significantly decreased tumor burden compared to carcinogen-exposed, but untreated animals. AFPep was active on oral administration in three different assays, in two different species, in both immature and adult animals, for the prevention as well as for therapy of breast cancer (30). Active oral doses were similar to those used for i.p. or s.c. administration, suggesting appreciable bioavailability for AFPep. Oral availability is an important consideration for a prospective agent that would be chronically administered.

Using standard screens for frank toxicity, high doses of AFPep were administered by bolus injection to groups of mice, or were given once daily for 5 days to groups of mice, and a number of endpoints were assessed. There were no indications of toxicity at any time, at any dose $(6,30)$. Other animals (rats and mice) were treated daily for various time periods with therapeutic or preventive (i.e., low) doses of AFPep, and again no evidence of toxicity was seen in any indicator. However, subtle but important endpoints that could assess potential side effects were not evaluated in the preliminary studies. To bring the drug forward for clinical testing, further assessments of side effects are needed.

Because of the desirability of using AFPep as a preventative as well as a therapeutic agent, there is need to investigate the effects of AFPep on the reproductive system. Although many breast cancer patients are post-menopausal and would not be affected by a disrupted menstrual cycle, there are women who are diagnosed with breast cancer at a younger age, and disruption of their fertility or menstrual cycle due to treatment may be undesirable. Especially if an available drug were completely non-toxic, women at a high risk for breast cancer may elect preventative treatment while they are premenopausal.

The estrous cycle in rodent reproduction is the equivalent in the rat of the human menstrual cycle. The phases of the cycle (estrus, proestrus, metestrus and diestrus) are regulated by a negative feedback mechanism involving estrogen, the hypothalamus and associated hormones. Because AFPep is an anti-estrogen, it is especially important to assess its effect on the rodent estrous cycle and fertility. While continuous monitoring of the hormone levels in serum would indicate cycle phase and estrous cycle progression, the method is burdensome and repeatedly invasive. However, tracking the estrous cycle by vaginal smears is relatively easy and very reliable $(31,32)$. Hence, five estrous cycles were monitored in rats chronically receiving AFPep (or tamoxifen or saline), and then fertility and fecundity were assessed in these same rats as measures of the effects of AFPep on the reproductive system.

\section{Materials and methods}

Chemicals. Tamoxifen (purity $>99 \%$, lot numbers $115 \mathrm{~K} 1068$, 062K0946 and 115K1068) was obtained from Sigma. Solutions were prepared in corn oil $1 \mathrm{~h}$ prior to use each day: $1.0 \mathrm{mg}$ of tamoxifen was weighed and dissolved in $4.0 \mathrm{ml}$ of corn oil. AFPep's linear pharmacophoric region (sequence TOVNO) (28) was prepared using automatic FMOC chemistry as described (29) on a Pioneer Peptide Synthesis System (PerSeptive Biosystem, Inc.). Purity was analyzed by mass spectroscopy. Peptide solutions were prepared in saline prior to use each day: $1.5 \mathrm{mg}$ of AFPep was weighed and dissolved in $3.0 \mathrm{ml}$ of saline and was used immediately after it was made.

Animals. Female Sprague-Dawley rats were acquired from Taconic Farms in Germantown, New York. The animals arrived at age 45 days and were allowed to adjust to the facility for five days. They were then handled daily for sixteen days prior to initiation of vaginal lavage to condition them to handling. All female rats were housed two per cage in plastic cages that were changed twice per week and stored in a temperature and humidity controlled room. They were maintained under a $12 \mathrm{~h}$ light-dark cycle with lights out at 7 p.m., and fed a standardized diet of RMH3000 and filtered water ad libitum. All animal husbandry was performed by female lab personnel and female animal facility employees. The cages were kept in a positive pressure room and no males (of any species) were allowed in the room during the estrous cycle investigation to avoid the influence of male hormones on the estrous cycle. Male rats obtained from Taconic Farms were housed singly for $24 \mathrm{~h}$ in a separate room to allow them to adjust to the animal facility.

\section{Methods}

Study groups. Thirty female rats were randomly assigned to one of three groups (control, tamoxifen-treated or AFPeptreated). The dose of tamoxifen $(50 \mu \mathrm{g} / \mathrm{rat} / \mathrm{day}$, in $0.2 \mathrm{ml})$ was chosen by converting the human daily prophylactic dose (20 mg) to the rat equivalent using a calculator provided by the Food and Drug Administration Center for Drug Evaluation 


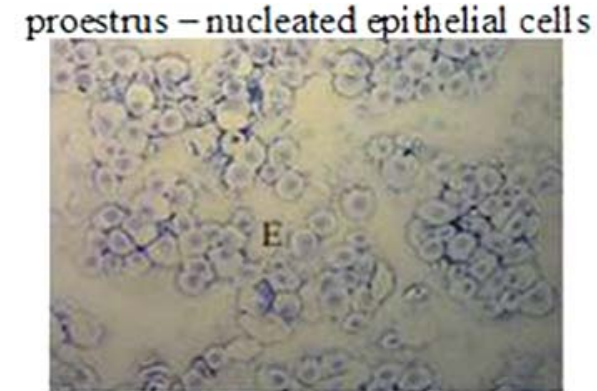

metestrus - pol ymorphonuclear leukocytes, keratinized cells, epithelial cells

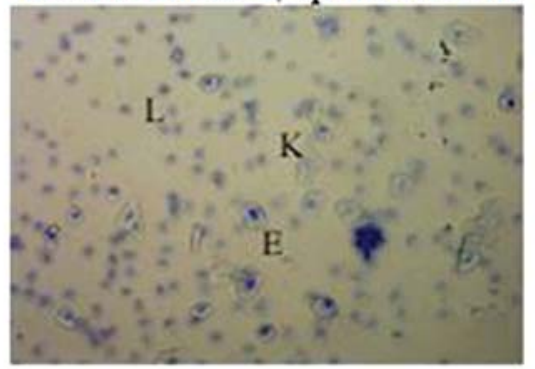

estrus - keratinized cells

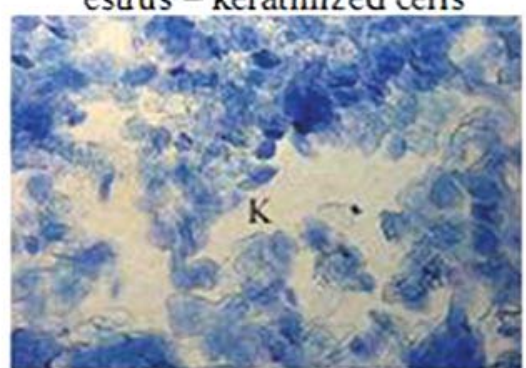

diestrus - polymorphonuclear leukocytes, viscous mucus (stained blue)

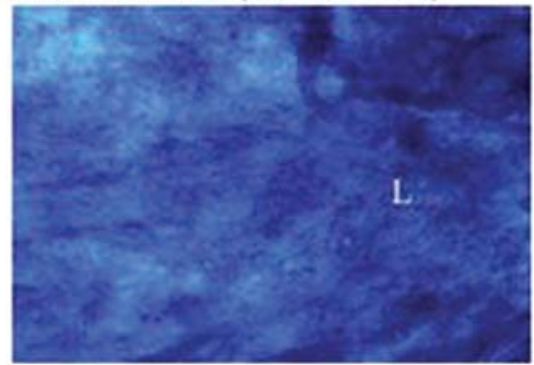

Figure 1. Cytology of vaginal smears. Vaginal smears were taken using a pipetter with plastic tips to aspirate $50 \mu 1 \mathrm{dH}_{2} \mathrm{O}$. Slides were air dried and stained using $0.1 \%$ Toluidine Blue to show nucleation. Slides are shown at magnification, x20. E, epithelial cell; K, keratinized cell and L, leukocyte (neutrophil).

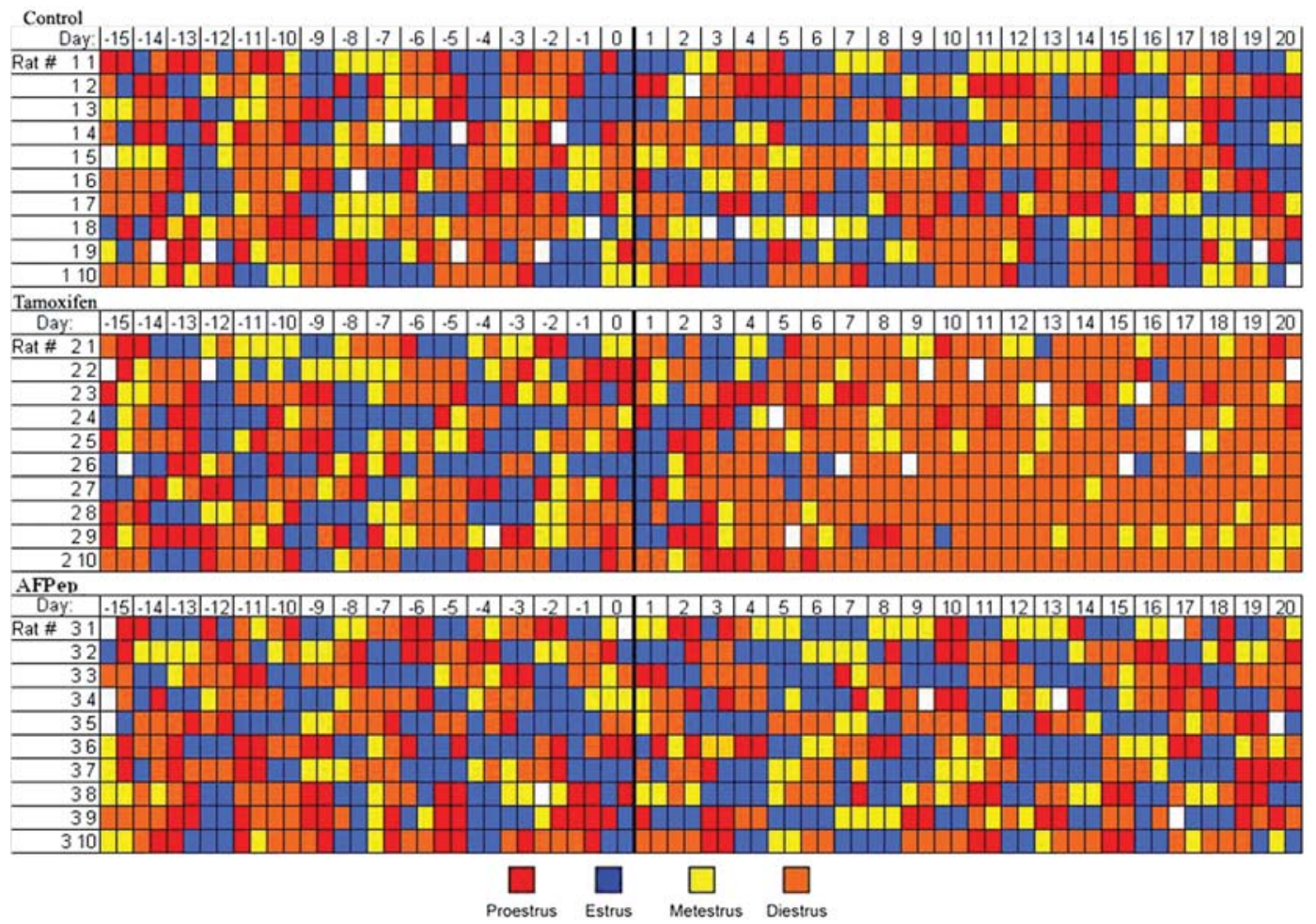

Figure 2. Estrous cycle phases of individual rats before and during treatment with tamoxifen or AFPep. Each row tracks the estrous cycle phases of one individual rat as determined by vaginal smear. Columns represent day of the experiment. The first column under each day is morning, the second is afternoon. Strong vertical line represents beginning of treatment. Tamoxifen group received $50 \mu \mathrm{g}$ of tamoxifen dissolved in $0.2 \mathrm{ml}$ corn oil by s.c. injection. The AFPep group received $100 \mu \mathrm{g}$ of peptide (TOVNO) dissolved in $0.2 \mathrm{ml}$ saline by s.c. injection. Red, p; blue, e; yellow, m and orange, d. Uncolored blocks represent unsuccessful smears.

and Research. AFPep dose (100 $\mu \mathrm{g} / \mathrm{rat} / \mathrm{day}$ in $0.2 \mathrm{ml})$ was chosen based on the dose-response curve of AFPep generated previously (6). All treatments were given subcutaneously on the back.
Estrous cycle monitoring. Procedures used were similar to those of Grasso et al (32-34) and Kim et al (31). Beginning at age 66 days, vaginal smears were obtained twice daily from all 30 animals (morning smears between 8 and 10 a.m. 
and afternoon smears between 2 and 4 p.m.). Vaginal smears were taken using plastic pipette tips to instill $50 \mu 1$ deionized water into the vagina of the rat, with care not to insert too far and stimulate the cervix. The aspirated water and vaginal secretion were applied to clean glass slides. Smears were dried and stained with $0.1 \%$ Toluidine Blue and viewed with a light microscope to observe cell populations and determine estrous cycle phase as in Fig. 1. After 16 days of vaginal smears prior to treatment (time for four complete cycles), animals (now age 81 days) were given daily injections of $0.2 \mathrm{ml}$ saline, $50 \mu \mathrm{g}$ tamoxifen in $0.2 \mathrm{ml}$ corn oil, or $100 \mu \mathrm{g}$ AFPep in $0.2 \mathrm{ml}$ saline. The daily injections were given following the afternoon smear. Treatments and vaginal smears were continued for 21 days.

Fertility assessment. After 21 days of treatment for the three groups, females were placed into cages with a male rat of proven fertility (two females and one male per cage). Females continued to receive AFPep, tamoxifen, or vehicle treatments as during estrous cycle monitoring. Each morning for two weeks, the females were checked for a vaginal plug. When no plug was present, a vaginal smear was taken and examined for the presence of sperm. On the day that either a plug or sperm was present (Gestation Day 0), females were separated from the male and maintained on treatment. At birth, pups were counted and weighed. Data collected included number of days before mating, number of rats that mated in each group, days before Gestation Day 0, successful pregnancy rate for each treatment group, length of gestation, number of pups per litter, and average body weight of pups.

Body weight of each adult female rat was measured to the nearest $0.5 \mathrm{~g}$ on the first day of vaginal smears, on the first day of injection, and on the final day of estrous cycle monitoring preceding initiation of fertility assessment.

Statistical analysis. Body weight and fertility data were compared across groups using Dunnett's test. Estrous cycle regularity was compared using a visual color-coded depiction (31), shown in Fig. 2. For each treatment group, the number of each of the colored boxes was counted and compared across groups using Dunnett's test. For each case, statistical significance was specified as being the $\mathrm{p}<0.05$ level.

\section{Results}

Fig. 1 shows photographs of vaginal smears that demonstrate the criteria used to determine cycle phase based on vaginal smear cytology. The proestrus smear is dominated by round cells that have blue-stained nuclei. These are epithelial cells, which degrade during estrus into the blue-stained irregularly shaped (keratinized) cells seen in the estrus smear. These epithelial cells are most prominent in the morning of proestrus. Estrogen is required for the maintenance of these epithelial cells, and the lower level of estrogen in the following phase (estrus) results in these cells being degraded into cornified, or keratinized, cells. Some keratinized cells are already visible in the afternoon of proestrus. During the day of estrus, the vaginal smear consists almost exclusively of keratinized epithelial cells. Following estrus is the 2-3 day diestrus phase, which is commonly divided into two phases: metestrus and diestrus. During this phase, vaginal smears are predominated by numerous leukocytes accompanied by an

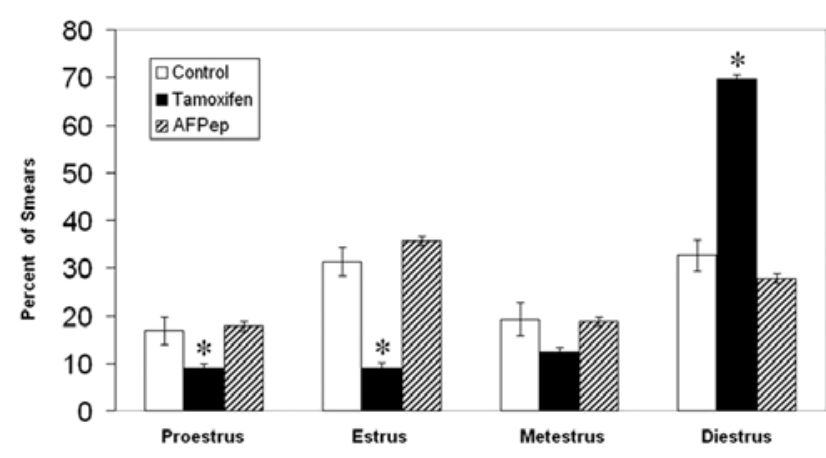

Figure 3. Incidence of estrous cycle phases during treatment with tamoxifen or AFPep. The graph is designed to facilitate comparisons across treatment groups, in which the bars represent incidence (\%) of each phase during treatment. Tamoxifen significantly increased the amount of diestrus and decreased the amount of proestrus and estrus. AFPep had no significant effect. *Statistically significant difference from control $(\mathrm{p}<0.05)$.

increasing number of nucleated cells and keratinized cells that are shrinking in size. In the metestrus smear, small round cells (polymorphonuclear leukocytes) that have stained a faint blue have invaded. There are also keratinized cells that are still degrading and some new epithelial cells forming. During diestrus, the smear is almost completely stained bright blue. This is the viscous mucus that is characteristic of this phase. Leukocytes are visible throughout the mucus, stained dark blue.

Estrous cycle. Estrous cycle data are displayed in Fig. 2 using a color code (30). Proestrus is coded red, estrus is coded blue, metestrus is coded yellow and diestrus is coded orange. Each rat is represented by a row in the Table and each block across the row represents one vaginal smear. This method of recording data provides a visual representation of phase incidence before and during treatment. The bold, vertical black line indicates when treatment with AFPep, tamoxifen or saline began. From this visual representation of the data, it is clear that in general, the rats were cycling in a smooth, regular pattern before treatment started. The slight cycle irregularities that are present in both saline-and AFPeptreated groups can be attributed to the fact that cycle phase was determined only twice daily, leaving more than $12 \mathrm{~h}$ unmonitored each night during which a rat may have begun and completed a phase. During treatment, the tamoxifentreated rats became locked in diestrus, while the AFPeptreated and saline-treated rats continued to cycle regularly. Quantitative evaluation is possible by analyzing the data in two ways: across treatments (i.e., comparing the tamoxifentreated group to the saline-treated group), or within a treatment (comparing the treatment period to the pre-treatment part of the study).

Across treatments. Fig. 3 depicts the incidence of each phase during the treatment period (days 1-20 of the experiment, while the animals were 81-101 days of age) for each group. During this time, the tamoxifen group displayed significantly more diestrus and significantly less estrus and proestrus than the saline-treated animals. Animals in the AFPep-treated group did not differ from the saline group in any phase. 


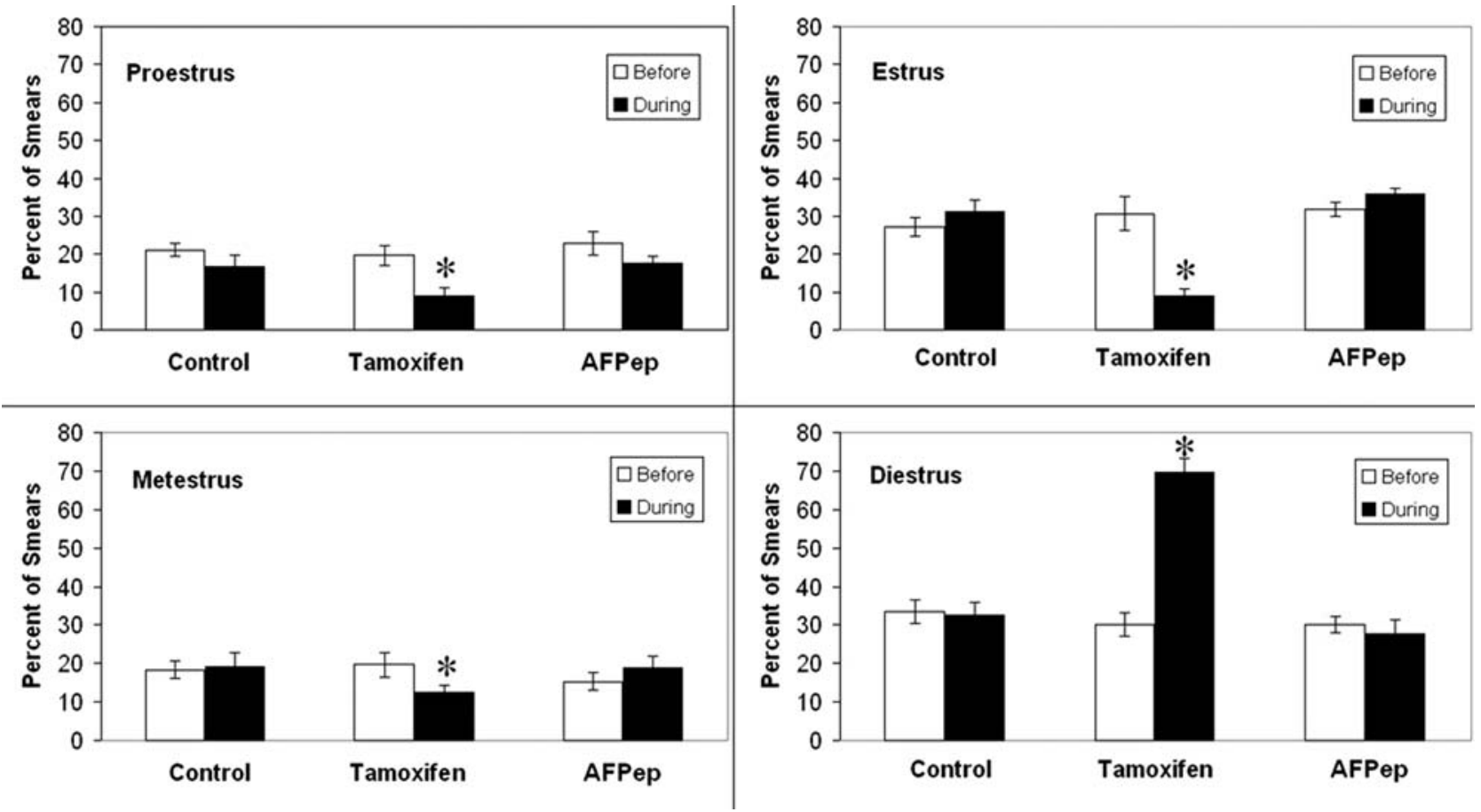

Figure 4. Comparison of incidence of each estrous cycle phase before and during treatment with tamoxifen or AFPep. The graph is designed to allow comparison of treatment to pre-treatment stages of the experiment. The series labeled 'Before' represents the incidence (\%) of proestrus, estrus, metestrus and diestrus, across the sixteen days prior to treatment in each group. The series labeled 'During' represents the incidence of proestrus, estrus, metestrus and diestrus, across the 20 days of treatment. Tamoxifen significantly increased the incidence of diestrus while decreasing the incidence of the other three phases. AFPep had no significant effect. *Statistically significant difference between before and after $(\mathrm{p}<0.05)$.

Within treatments. Fig. 4 compares the change in incidence of each phase from before treatment to during treatment for each of the groups. The change was significant for all four phases in the tamoxifen treated group and there were no significant changes in the saline- or AFPep-treated groups.

Fertility. After five complete cycles (21 days), male rats were introduced while females continued treatment with AFPep or tamoxifen. Data on mating behaviors and pregnancy are tabulated in Table I. Tamoxifen had an inhibitory effect on fertility and mating behavior, while AFPep had no apparent effect. In both the control animals and the AFPep-treated animals, eight of ten rats mated within four days (one cycle), as determined by the presence of sperm in the vaginal smear. Only one of the tamoxifen-treated rats mated within this time. After two weeks, only one rat from the control group and one rat from the AFPep-treated group failed to mate, while half of the tamoxifen treated rats did not mate. The average number of days before Gestation Day 0 (number of days it took the rats to mate) was not significantly different in the rats that did mate within the two-week time limit. Six of the nine rats that mated in the control group gave birth and all pups were healthy. Eight of the nine rats that mated in the AFPep group gave birth to pups, all of which were healthy. While this study was not designed to monitor teratogenicity, it was clear that there were no gross pathologies in any of the pups in either the AFPep or saline groups. The length of gestation, number of pups and pup body weights were all similar in the saline- and AFPep-treated groups. Tamoxifen, on the other hand, disrupted the estrous cycle to the extent that none of the treated rats became pregnant.

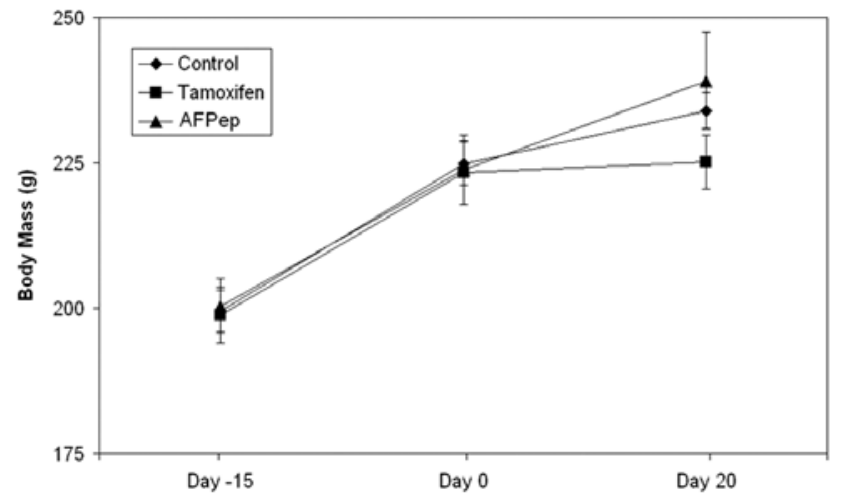

Figure 5. Effect of tamoxifen or AFPep on body mass. Neither tamoxifen nor AFPep had a significant effect on body mass, but tamoxifen decreased the rate of weight gain.

None gave birth, and upon dissection, none had signs of pregnancy.

Body mass. AFPep had no significant effect on body mass (Fig. 5), as was previously reported $(5,6,30)$. Although tamoxifen has been shown previously to decrease body mass significantly in rats $(31,35)$, the absolute difference in body weight did not achieve statistical significance at any time. However, the growth rate of rats during treatment with tamoxifen $(0.09 \mathrm{~g} /$ day $)$ was significantly slower than the rate in the control group $(0.455 \mathrm{~g} / \mathrm{day}) \mathrm{p}<0.04$. In the AFPeptreated group the growth rate was not different from that of the control animals. 
Table I. Effects of treatment with tamoxifen or AFPep on reproductive performance.

\begin{tabular}{|c|c|c|c|c|c|c|c|}
\hline Treatment & $\begin{array}{l}\text { Number } \\
\text { that mated } \\
\text { in } \leq 4 \text { days }\end{array}$ & $\begin{array}{c}\text { Total } \\
\text { number } \\
\text { that mated }^{\mathrm{a}}\end{array}$ & $\begin{array}{c}\text { Days before } \\
\text { GD0 }^{\mathrm{b}}\end{array}$ & $\begin{array}{l}\text { Number } \\
\text { that gave } \\
\text { birth }\end{array}$ & $\begin{array}{l}\text { Length of } \\
\text { gestation } \\
\text { (days) }\end{array}$ & $\begin{array}{c}\text { Number of } \\
\text { pups }\end{array}$ & $\begin{array}{c}\text { Pup BWc } \\
\text { (g) }\end{array}$ \\
\hline Saline & 8 & 9 & $\begin{array}{c}3.4 \pm 1.24 \\
n=9\end{array}$ & 6 & $\begin{array}{c}21.7 \pm 0.18 \\
n=6\end{array}$ & $\begin{array}{c}11.2 \pm 0.79 \\
\mathrm{n}=6\end{array}$ & $\begin{array}{c}6.4 \pm 0.20 \\
n=6\end{array}$ \\
\hline Tamoxifen & $1^{\mathrm{f}}$ & $5^{\mathrm{d}}$ & $\begin{array}{c}6.2 \pm 1.96 \\
n=5\end{array}$ & $0^{\mathrm{e}}$ & - & - & - \\
\hline AFPep & 8 & 9 & $\begin{array}{c}2.7 \pm 0.44 \\
n=9\end{array}$ & 8 & $\begin{array}{c}22.1 \pm 0.23 \\
n=8\end{array}$ & $\begin{array}{c}10 \pm 1.04 \\
n=8\end{array}$ & $\begin{array}{c}6.8 \pm 0.17 \\
n=8\end{array}$ \\
\hline
\end{tabular}

Values for days before GD0, length of gestation, number of pups and Pup BW are mean \pm SE (initial n=10 animals per treatment group). aTotal number that mated within two weeks; ${ }^{\mathrm{b}} \mathrm{GD} 0$, Gestation Day 0 (number of days after introduction of male before evidence of mating); ${ }^{\mathrm{C}} \mathrm{BW}$, average body weight of pups in each litter the day of birth; ${ }^{\mathrm{d}}$ Statistically significant difference from control $(\mathrm{p}<0.05)$; ${ }^{\mathrm{e}}(\mathrm{p}<0.01)$; ${ }^{\mathrm{f}}(\mathrm{p}<0.001)$. Statistics calculated using $\chi^{2}$ tests.

\section{Discussion}

The results of this study demonstrate that the estrous cycle, fertility and fecundity of the rat are not disrupted by AFPep. This is different from the action of tamoxifen, which substantially disrupts these reproductive functions. This is critical for the development of AFPep as a chemopreventive, as well as a therapeutic, agent for breast cancer in women. Ideally in chemoprevention, one would require efficacy without toxicity for an agent that is being given to women who are presumably healthy. AFPep has efficacy without toxicity in all studies to date, including this study which showed that daily injection of AFPep did not disrupt reproductive function in rats.

This is a seminal finding about AFPep in that its parent protein, AFP, grossly disrupts reproductive tissues and function. Aussel et al (17) have shown that injection of AFP into rats resulted in decreased ovarian activity as evidenced by a decrease in the number of ovarian follicles and corpora lutea. Sparks et al (36) showed that injection of AFP into mice suppressed $\mathrm{E}_{2}$-induced proliferation of vaginal epithelium. Pool et al (18) found that rats bearing AFP-secreting hepatomas were arrested in diestrus, an observation which was supported in studies by Nerad et al (20) and Couinaud et al (19) in humans. The latter investigators observed amenorrhea in women with hepatoma, and noted that amenorrhea resolved after resection of the AFP-secreting tumor. The fact that no disruption of the estrous cycle was seen in rats treated with AFPep suggests that in isolating the anti-oncogenic site of AFP (i.e., developing AFPep), the epitope(s) of AFP that adversely impact the reproductive system were effectively parsed out.

Tamoxifen altered the estrous cycle in this study, causing persistent diestrus. This is in agreement with the studies of Orcheson et al (37) and Kim et al (31). It is noteworthy that tamoxifen was originally developed by Harper and Walpole (38) in the 1960s intended as a birth control agent for preventing postcoital implantation in rats, but did not advance to a 'morning after pill' in humans because of its side effects. Its 'rescue' as a drug followed the finding by Jordan $(39,40)$ that tamoxifen is a very effective agent for the treatment and prevention of breast cancer and it has been developed successfully for use in humans for this purpose $(1,41)$. Women have been observed to achieve pregnancy while on tamoxifen (42) and tamoxifen can have harmful effects on the fetus (42), so although its risk/benefit ratio is justified for breast cancer (warning women not to become pregnant while on tamoxifen), it it is not useful as a birth control agent. Other side effects which call into question its risk/benefit ratio for non-cancer patients are nausea, hot flashes, weight gain, fluid retention, vision changes and vaginal discharge. Less frequent but more serious problems are uterine hyperplasia which can progress to uterine cancer, and thromboembolic episodes which can progress to deep vein thrombosis, occurring in less than $1 \%$ of patients receiving tamoxifen $(1,41)$. This obviously gives pause when considering its use for chemoprevention in otherwise healthy women deemed to be at elevated risk for breast cancer. No side effects have as yet been seen with AFPep, and in experimental animals, AFPep has anti-breast cancer effects equivalent to those of tamoxifen (4). This may be due to the fact that AFPep has a mechanism quite different from that of tamoxifen and other SERMs. AFPep is a multikinase inhibitor that tamps down phosphorylation of the estrogen receptor, FAK and c-kit, and impedes the growth stimulatory signals mediated by these transducers (30). AFPep seems to express this outcome more prominently in cancer tissues than in normal tissues $(5,30)$. Tamoxifen, on the other hand, is a partial agonist for the estrogen receptor which induces growth inhibition in the breast but growth stimulation in other tissues such as the uterus.

The over-reaching goal of chemoprevention is to develop agents that have efficacy without toxicity. Peptide drugs that are analogs of naturally occurring proteins may be ideal lead compounds for achieving this goal, especially given that the ability to isolate a single active site may obviate side effects 
while maintaining efficacy. Metabolites of peptides would be simple amino acids, obviating toxicity. Previous studies suggest that AFPep would be an effective preventive agent. The results from this animal study suggest that it would also be safe, without side effects on reproduction. If these results translate successfully to human trials, many at-risk women would be able to prevent breast cancer with minimal, if any, morbidity.

\section{Acknowledgements}

Support by NIH 5 R01 CA 102540, 5 R25 GM 062460, the Alpha Omega Alpha Carolyn L. Kuckein Student Research Fellowship (AMT), and the Samuel Powers M.D. Research Fellowship (AMT).

\section{References}

1. Fisher B, Costantino JP, Wickerham DL, et al: Tamoxifen for prevention of breast cancer: report of the National Surgical Adjuvant Breast and Bowel Project P-1 Study. J Natl Cancer Inst 90: 1371-1388, 1998.

2. Kraft JK and Hughes T: Polypoid endometriosis and other benign gynaecological complications associated with Tamoxifen therapy - a case to illustrate features on magnetic resonance imaging. Clin Radiol 61: 198-201, 2006.

3. MacGregor JI and Jordan VC: Basic guide to the mechanisms of antiestrogen action. Pharmacol Rev 50: 151-196, 1998.

4. Andersen TT, Georgekutty J, DeFreest LA, et al: An alphafetoprotein-derived peptide reduces the uterine hyperplasia and increases the antitumour effect of tamoxifen. Br J Cancer 97: 327-333, 2007

5. Bennett JA, Mesfin FB, Andersen TT, Gierthy JF and Jacobson HI: A peptide derived from alpha-fetoprotein prevents the growth of estrogen-dependent human breast cancers sensitive and resistant to tamoxifen. Proc Natl Acad Sci USA 99: 2211-2215, 2002.

6. Parikh RR, Gildener-Leapman N, Narendran A, et al: Prevention of N-methyl-N-nitrosourea-induced breast cancer by alpha-fetoprotein (AFP)-derived peptide, a peptide derived from the active site of AFP. Clin Cancer Res 11: 8512-8520, 2005 .

7. Jacobson HI, Thompson WD and Janerich DT: Multiple births and maternal risk of breast cancer. Am J Epidemiol 129: 865-873, 1989.

8. Richardson BE, Hulka BS, Peck JL, Hughes CL, van den Berg BJ, Christianson RE and Calvin JA: Levels of maternal serum alpha-fetoprotein (AFP) in pregnant women and subsequent breast cancer risk. Am J Epidemiol 148: 719-727, 1998.

9. Sonnenschein C, Ucci AA and Soto AM: Growth inhibition of estrogen-sensitive rat mammary tumors. Effect of an alphafetoprotein-secreting hepatoma. J Natl Cancer Inst 64: 1147-1152, 1980.

10. Sonnenschein C, Ucci AA and Soto AM: Age-dependent growth inhibition of estrogen-sensitive rat mammary tumors. Probable role of alpha-fetoprotein. J Natl Cancer Inst 64: 1141-1146, 1980.

11. Terentiev AA and Moldogazieva NT: Structural and functional mapping of alpha-fetoprotein. Biochemistry 71: 120-132, 2006.

12. Gately S, Twardowski P, Stack MS, et al: The mechanism of cancer-mediated conversion of plasminogen to the angiogenesis inhibitor angiostatin. Proc Natl Acad Sci USA 94: 10868-10872, 1997

13. Sasaki T, Fukai N, Mann K, Gohring W, Olsen BR and Timpl R: Structure, function and tissue forms of the C-terminal globular domain of collagen XVIII containing the angiogenesis inhibitor endostatin. EMBO J 17: 4249-4256, 1998.

14. Semeniuk DJ, Boismenu R, Tam J, Weissenhofer W and Murgita RA: Evidence that immunosuppression is an intrinsic property of the alpha-fetoprotein molecule. Adv Exp Med Biol 383: 255-269, 1995.
15. Lin Y, Jin DK, Vacher J and Feuerman MH: Sequence requirements for alpha-fetoprotein gene expression during liver regeneration. Cell Growth Differ 6: 1549-1558, 1995.

16. Mizejewski GJ: Biological roles of alpha-fetoprotein during pregnancy and perinatal development. Exp Biol Med 229: 439-463, 2004.

17. Aussel C, Lafaurie M, Masseyeff $\mathrm{R}$ and Stora C: In vivo regulation of ovarian activity by alpha-fetoprotein. Steroids 38 : 195-204, 1981

18. Pool TB, Hagino N and Cameron IL: Relationship between functional castration and alpha-fetoprotein produced by hepatoma-bearing female rats. J Reprod Fertil 53: 39-44, 1978.

19. Couinaud C, Schwarzmann V, Ceoara B, Orengo P and Fitterer R: Malignant hepatoma with amenorrhea and galactorrhea. Disappearance of the endocrine syndrome following right hepatectomy. Ann Chir 27: 151-156, 1973.

20. Nerad V, Brzek V, Skaunic V and Kopecny J: Secondary amenorrhea, the first symptom of hepatoma. Collection of the Scientific Works of the Medical School of Charles University, Prague 12: 257-262, 1969

21. Festin SM, Bennett JA, Fletcher PW, Jacobson HI, Shaye DD and Andersen TT: The recombinant third domain of human alpha-fetoprotein retains the antiestrotrophic activity found in the full-length molecule. Biochim Biophys Acta 1427: 307-314, 1999.

22. Morinaga T, Sakai M, Wegmann TG and Tamaoki T: Primary structures of human alpha-fetoprotein and its mRNA. Proc Natl Acad Sci USA 80: 4604-4608, 1983.

23. Eisele LE, Mesfin FB, Bennett JA, et al: Studies on analogs of a peptide derived from alpha-fetoprotein having antigrowth properties. J Pept Res 57: 539-546, 2001.

24. Eisele LE, Mesfin FB, Bennett JA, et al: Studies on a growthinhibitory peptide derived from alpha-fetoprotein and some analogs. J Pept Res 57: 29-38, 2001.

25. Mizejewski GJ, Dias JA, Hauer CR, Henrikson KP and Gierthy J: Alpha-fetoprotein derived synthetic peptides: assay of an estrogen-modifying regulatory segment. Mol Cell Endocrinol 118: 15-23, 1996.

26. Mesfin FB, Bennett JA, Jacobson HI, Zhu S and Andersen TT: Alpha-fetoprotein-derived antiestrotrophic octapeptide. Biochim Biophys Acta 1501: 33-43, 2000.

27. DeFreest LA, Mesfin FB, Joseph L, et al: Synthetic peptide derived from alpha-fetoprotein inhibits growth of human breast cancer: investigation of the pharmacophore and synthesis optimization. J Pept Res 63: 409-419, 2004.

28. Kirschner KN, Lexa KW, Salisburg AM, et al: Computational design and experimental discovery of an antiestrogenic peptide derived from alpha-fetoprotein. J Am Chem Soc 129: 6263-6268, 2007.

29. Mesfin FB, Andersen TT, Jacobson HI, Zhu S and Bennett JA: Development of a synthetic cyclized peptide derived from alpha-fetoprotein that prevents the growth of human breast cancer. J Pept Res 58: 246-256, 2001

30. Bennett JA, Defreest L, Anaka I, Saadati H, Balulad S, Jacobson HI and Andersen TT: AFPep: an anti-breast cancer peptide that is orally active. Breast Cancer Res Treat 98: 133-141, 2006.

31. Kim HS, Shin JH, Moon HJ, et al: Evaluation of the 20-day pubertal female assay in Sprague-Dawley rats treated with DES, tamoxifen, testosterone, and flutamide. Toxicol Sci 67: 52-62, 2002.

32. Grasso P, Rozhavskaya-Arena M and Reichert LE Jr: Cysteine residues in a synthetic peptide corresponding to human folliclestimulating hormone beta-subunit receptor-binding domain 8195 [hFSH-beta-(81-95)] modulate the in vivo effects of hFSHbeta-(81-95) on the mouse estrous cycle. Regul Pept 81: 67-71, 1999.

33. Grasso P, Rozhavskaya M and Reichert LE Jr: In vivo effects of human follicle-stimulating hormone-related synthetic peptide hFSH-beta-(81-95) and its subdomain hFSH-beta-(90-95) on the mouse estrous cycle. Biol Reprod 58: 821-825, 1998.

34. Grasso P and Reichert LE Jr: In vivo effects of folliclestimulating hormone-related synthetic peptides on the mouse estrous cycle. Endocrinology 137: 5370-5375, 1996.

35. Peterson NC, Servinsky MD, Christian A, et al: Tamoxifen resistance and Her2/neu expression in an aged, irradiated rat breast carcinoma model. Carcinogenesis 26: 1542-1552, 2005.

36. Sparks RL and Grubbs BG: Suppression of estrogen action on mouse vagina by serum containing alpha-fetorprotein. Anat Rec 193: 689-690, 1979. 
37. Orcheson LJ, Rickard SE, Seidl MM and Thompson LU: Flaxseed and its mammalian lignan precursor cause a lengthening or cessation of estrous cycling in rats. Cancer Lett 125: 69-76, 1998.

38. Harper MJ and Walpole AL: A new derivative of triphenylethylene: effect on implantation and mode of action in rats. J Reprod Fertil 13: 101-119, 1967.

39. Jordan VC: Targeted antiestrogens to prevent breast cancer. Trends Endocrinol Metab 10: 312-317, 1999.
40. Jordan VC: Tamoxifen: Catalyst for the change to targeted therapy. Eur J Cancer 44: 30-38, 2008.

41. Fisher B, Costantino J, Redmond C, et al: A randomized clinical trial evaluating tamoxifen in the treatment of patients with nodenegative breast cancer who have estrogen-receptor-positive tumors. N Engl J Med 320: 479-484, 1989.

42. Barthelmes L and Gateley CA: Tamoxifen and pregnancy. Breast 13: 446-451, 2004. 